\title{
Cognitive deficits in non-demented diabetic elderly appear independent of brain amyloidosis
}

Gloria C. Chiang, M.D, ${ }^{1}$ Eileen Chang, B.A., ${ }^{1}$ Sneha Pandya, M.S., ${ }^{1}$ Amy Kuceyeski, Ph.D., ${ }^{1}$ James Hu, B.A., ${ }^{1}$ Richard Isaacson, M.D. ${ }^{2}$ Christine Ganzer, Ph.D., ${ }^{3}$ Aaron Schulman, M.D., ${ }^{4}$ Vivian Sobel, M.D., ${ }^{4}$ Shankar Vallabhajosula, Ph.D. ${ }^{1}$ for the Alzheimer's Disease Neuroimaging Initiative, ${ }^{*}$ and Lisa Ravdin, Ph.D. ${ }^{2}$

${ }^{1}$ From the Department of Radiology, Division of Neuroradiology, Weill Cornell Medical College, NewYork-Presbyterian Hospital, New York, NY

${ }^{2}$ From the Department of Neurology, Weill Cornell Medical College, NewYork-

Presbyterian Hospital, New York, NY

${ }^{3}$ From the Department of Nursing, Hunter-Bellevue School of Nursing, New York, NY

${ }^{4}$ From the Department of Medicine, Division of Endocrinology, Weill Cornell Medical College, NewYork-Presbyterian Hospital, New York, NY

*Data used in preparation of this article were obtained from the Alzheimer's Disease Neuroimaging Initiative (ADNI) database (adni.loni.usc.edu). As such, the investigators within the ADNI contributed to the design and implementation of ADNI and/or provided data but did not participate in analysis or writing of this report. A complete listing of ADNI investigators can be found at: http://adni.loni.usc.edu/wp-

content/uploads/how_to_apply/ADNI_Acknowledgement_List.pdf

\section{Corresponding author:}

Gloria C. Chiang, M.D.

Department of Radiology, Division of Neuroradiology

Weill Cornell Medical College, NewYork-Presbyterian Hospital

525 East 68th Street Box 141, Starr Pavilion

New York, NY 10065

Phone: $212-746-2616$

Fax: 212-746-8597

email: gcc9004@med.cornell.edu

Keywords: beta-amyloid, cognition, Type 2 diabetes, positron emission tomography

\section{Grant Support:}

This work was supported by an NIH National Center for Advancing Translational Sciences/CTSC grant (UL1 TR000457-06)

Data collection and sharing for this project was funded by the Alzheimer's Disease Neuroimaging Initiative (ADNI) (National Institutes of Health Grant U01 AG024904) and DOD ADNI (Department of Defense award number W81XWH-12-2-0012). ADNI is funded by the National Institute on Aging, the National Institute of Biomedical Imaging and Bioengineering, and through generous contributions from the following: Alzheimer's Association; Alzheimer's Drug Discovery Foundation; Araclon Biotech; BioClinica, Inc.; 
Biogen Idec Inc.; Bristol-Myers Squibb Company; Eisai Inc.; Elan Pharmaceuticals, Inc.; Eli Lilly and Company; Eurolmmun; F. Hoffmann-La Roche Ltd and its affiliated company Genentech, Inc.; Fujirebio; GE Healthcare; ; IXICO Ltd.; Janssen Alzheimer Immunotherapy Research \& Development, LLC.; Johnson \& Johnson Pharmaceutical Research \& Development LLC.; Medpace, Inc.; Merck \& Co., Inc.; Meso Scale Diagnostics, LLC.; NeuroRx Research; Neurotrack Technologies; Novartis Pharmaceuticals Corporation; Pfizer Inc.; Piramal Imaging; Servier; Synarc Inc.; and Takeda Pharmaceutical Company. The Canadian Institutes of Health Research is providing funds to support ADNI clinical sites in Canada. Private sector contributions are facilitated by the Foundation for the National Institutes of Health (www.fnih.org). The grantee organization is the Northern California Institute for Research and Education, and the study is coordinated by the Alzheimer's Disease Cooperative Study at the University of California, San Diego. ADNI data are disseminated by the Laboratory for Neuro Imaging at the University of Southern California. 


\begin{abstract}
:
Background: To determine the effects of Type 2 diabetes (DM2) on levels of brain amyloidosis and cognition in a community-dwelling cohort of nondemented elderly individuals.
\end{abstract}

Methods: 33 subjects (16 DM2, 17 nondiabetic) were prospectively recruited. Subjects underwent a PET scan using the amyloid tracer, Pittsburgh Compound B, and a neuropsychological evaluation. Associations between DM2, brain amyloidosis, and cognition were assessed using multivariate regressions, adjusting for age and APOE4 status.

Results: DM2 subjects had lower global cognitive function $(p=0.018)$, as measured by the Repeatable Battery for the Assessment of Neuropsychological Status. There was no difference in brain amyloidosis between groups $(p=0.25)$.

Conclusions: Community-dwelling, nondemented individuals with DM2 had greater cognitive deficits, which do not appear to be mediated by brain amyloidosis. Further studies exploring potential mediators of these cognitive deficits should be performed. 


\section{Introduction:}

Both Type 2 diabetes (DM2) and Alzheimer's disease (AD) are major public health problems and sources of morbidity among older adults. With the burgeoning obesity epidemic, it is estimated that 285 million people worldwide carry a diagnosis of DM2. ${ }^{1}$ Thirty-four million people are estimated to suffer from dementia, with $70 \%$ of these cases attributable to AD. ${ }^{2}$ Many epidemiologic studies have found that DM2 increases the risk of developing dementia. ${ }^{3-7}$ However, the degree to which this increased risk is related to underlying AD pathology, such as brain amyloidosis, is unknown.

The pathological hallmark of $A D$ is brain amyloidosis on autopsy. ${ }^{8}$ Some have hypothesized that hyperglycemia in DM2 patients could prompt formation of advanced glycation endproducts, ${ }^{9}$ leading to increased beta-amyloid plaque aggregation in the brain. ${ }^{10-12}$ Others have hypothesized that high plasma insulin levels among DM2 subjects, secondary to insulin resistance at the tissue level, result in greater diversion of the insulin-degrading enzyme from its usual function of degrading amyloid, ${ }^{13}$ thereby increasing brain amyloidosis. However, two prior retrospective studies have not found increased brain amyloidosis in DM2. ${ }^{13,14}$ Possible reasons these studies did not find increase brain amyloidosis could be that they included cohorts with relatively mild DM2 disease, ${ }^{13}$ elderly subjects who already had age-related amyloid deposition, ${ }^{14}$ and mixed cohorts including subjects with mild cognitive impairment $(\mathrm{MCl})$ and $A D,{ }^{14}$ making it difficult to separate out the effects of DM2.

Since it is estimated that brain amyloidosis begins approximately $15-20$ years before onset of cognitive symptoms, ${ }^{15}$ we aimed to enroll a younger cohort of DM2 individuals than previously reported. Furthermore, it has been reported that diabetic patients with longer disease duration, greater severity of disease, and diabetic complications have a higher rate of cognitive impairment, ${ }^{16}$ so these high-risk individuals were enrolled into our study. Finally, we enrolled subjects who did not meet criteria for mild cognitive impairment $(\mathrm{MCl})$ or $A D$ to exclude potential confounding by pre-existing AD pathology, irrespective of the effects of DM2.

The purpose of this study, therefore, was to determine the effects of DM2 on brain amyloid deposition in vivo in a prospectively-enrolled, nondemented cohort, using positron emission tomography (PET) with the amyloid tracer, Pittsburgh Compound $B$ (PiB). Furthermore, we evaluated the association between DM2 and cognitive deficits, investigating potential mediators of disease.

\section{Materials and methods:}

\subsection{Subjects}

We prospectively enrolled 33 subjects in this study: 16 with diagnosed DM2 and 17 non-diabetic age-matched cognitively normal individuals. Individuals were recruited through flyers posted in the community, newspaper advertisements, and ambulatory care clinics. All subjects gave written informed consent for participation in this study, and this study was approved by the Institutional Review Board.

In order to target subjects with long-standing or severe diabetes, who may have the greatest risk of developing $A D,{ }^{16}$ inclusion criteria for DM2 subjects included at least one of the following: (1) DM2 diagnosed 10 or more years ago, (2) recent $\mathrm{HbA} 1 \mathrm{c}$ level of $7 \%$ or greater, (3) history of a DM2-related complication, such as retinopathy, nephropathy, or neuropathy, or (4) recently reported blood glucose levels of greater than $300 \mathrm{mg} / \mathrm{dl}$. 
All subjects were between the ages of 55 and 75, lived independently in the community, and were able to perform all routine activities of daily living. Subjects were excluded if they had significant comorbid medical conditions that could impact brain function, including major psychiatric disorders (i.e. major depression, bipolar disorder, and psychosis), brain tumors, prior strokes, significant traumatic brain injury (defined as requiring a visit to the emergency department or a hospital stay), seizure disorders, recent illicit drug use, alcohol abuse, and major medical problems, such as heart failure, recent myocardial infarction, renal failure, liver disease, chronic obstructive pulmonary disease, and malignancy. Medical records were also reviewed for exclusionary criteria.

\subsection{Clinical data}

To determine what aspect of DM2 may be related to brain amyloidosis or cognition, all subjects completed detailed questionnaires about their medical history, and medical records were also examined. Clinical data included duration of disease, recent hemoglobin A1c values, recent blood glucose readings, medications used to control DM2, and presence of diabetic complications, such as retinopathy, neuropathy, or nephropathy.

Clinical data for all subjects included recent weight and height, cholesterol levels, blood pressure measurements, smoking history, and exercise regimens, since these variables have been reported as affecting for AD. ${ }^{17,18}$ We also elicited a family history of dementia, since genetics could explain increased brain amyloidosis in otherwise cognitively normal individuals. ${ }^{19}$

Subjective memory complaints were elicited by the questions, "are you forgetful?" and "do you have difficulty remembering things?" Subjective memory complaints were further assessed by the Cognitive Change Index, ${ }^{20}$ Patients with subjective memory complaints, despite normal cognition on objective measures, have been shown to have greater longitudinal cognitive decline. ${ }^{21,22}$ Finally, since health literacy has been shown to affect health outcomes, including complications of DM2, all subjects also completed a health literacy questionnaire called the Short Test of Functional Health Literacy in Adults (STOFHLA). ${ }^{23,24}$

\subsection{Cognitive battery}

Cognitive testing was performed by the Director of Neuropsychology at our institution with 20 years of experience in evaluating patients with memory disorders. Cognitive assessment was performed using the Repeatable Battery for the Assessment of Neuropsychological Status (RBANS), which includes measures for immediate and delayed memory, visuospatial and constructional function, attention, and language ${ }^{25}$ Briefly, immediate and delayed memory were tested using tasks that involved recalling a list of words and a short story. Visuospatial and constructional function was tested using figure copying and line orientation tasks. Attention was tested using digit span and coding tasks. Language was tested using picture naming and semantic fluency tasks. The RBANS has been found to have almost $90 \%$ accuracy for discriminating between cognitive normal individuals and those with mild cognitive impairment. ${ }^{25}$ Cognitive processing speed and executive function were also evaluated using the Trail Making Test, ${ }^{26,27}$ with Part A measuring visual scanning and motor speed and Part B measuring executive function related to mental sequencing. Patients were also screened for comorbid depression and anxiety using the Beck Depression Inventory- $\mathrm{II}^{28}$ and the Beck Anxiety Inventory ${ }^{29}$ and excluded as necessary.

\subsection{APOE genotyping}


Blood was drawn from all subjects to isolate DNA for APOE genotyping, which was performed using polymerase chain reaction amplification, allele-specific primers, and identification of fragments on an agarose gel. ${ }^{30}$

\subsection{PiB PET Image Acquisition, Template Creation, and Analysis}

All subjects underwent an amyloid PET scan on a Siemens Biograph PET-CT scanner [Siemens, Knoxville, TN; $1 \mathrm{~mm}$ FWHM, $25 \mathrm{~cm}$ FOV] using a standardized research protocol. ${ }^{19}$ Briefly, all patients received an intravenous catheter for injection of $15 \mathrm{mCi}$ of $\mathrm{PiB}$. Sixty minutes after injection, subjects were scanned for 30 minutes with their eyes open in a quiet, dimly lit room. A low-dose CT scan was acquired for attenuation correction, and all images were reconstructed into a 512 × 512 matrix.

Summed PET images corresponding to the 60-90 minutes of PiB data were generated and nonlinearly normalized to a PiB template. The PiB template was generated by averaging the summed images of 48 cognitively normal individuals in the same age range, which were downloaded from the Alzheimer's Disease Neuroimaging Initiative (ADNI) online data repository. Orientation and origin for all the PiB PET images were automatically fixed to the anterior commissure to match the templates used in Statistical Parametric Mapping (SPM, Wellcome Trust Center for Neuroimaging), since SPM's "normalize" function uses the origin as a starting estimate. These reoriented PiB PET images and the mean PiB template were skull-stripped with the Brain Extraction Tool from FMRIB Software Library (FSL) ${ }^{31}$ to avoid any bias induced by skull staining. All the skull-stripped PiB PET images were then nonlinearly warped to the skull-stripped mean $\mathrm{PiB}$ template. Gray matter regions were parcellated using the Automated Anatomical Labeling (AAL) atlas to obtain 116 automated regions-of-interest. ${ }^{32}$ Regional PiB uptake values were then normalized by the subject's cerebellar reference uptake.

The ADNI is a longitudinal, multicenter, observational cohort study, which was designed to identify imaging and biochemical biomarkers for diagnosis and monitoring of AD. ${ }^{33}$ The ADNI was launched in 2003 by the National Institute on Aging (NIA), the National Institute of Biomedical Imaging and Bioengineering (NIBIB), the Food and Drug Administration (FDA), private pharmaceutical companies, and non-profit organizations, as a public-private partnership. The primary goal of ADNI has been to test whether serial magnetic resonance imaging (MRI), positron emission tomography (PET), other biological markers, and clinical and neuropsychological assessment can be combined to measure the progression of mild cognitive impairment $(\mathrm{MCl})$ and early Alzheimer's disease $(A D)$. For up-to-date information, please see www.adni-info.org.

Based on previously publications from researchers from the ADNI, it was determined that the 4 large regions of the brain that are most useful in measuring the degree of brain amyloidosis are the frontal region, anterior/posterior cingulate region, lateral parietal region, and lateral temporal region, using the cerebellum as a reference region. ${ }^{34,35}$ Using regions-of-interest from the AAL atlas, amyloid deposition in the frontal region was determined by averaging the bilateral superior frontal, bilateral superior orbital frontal, bilateral middle frontal, bilateral inferior frontal opercular, bilateral inferior frontal triangularis, bilateral supplemental motor, bilateral medial superior frontal, and bilateral middle orbital frontal regions of the brain. The cingulate region included the bilateral anterior, middle, and posterior cingulum regions. The lateral parietal region included the bilateral superior and inferior parietal regions, as well as the precuneus. The lateral temporal region included the bilateral and superior middle temporal regions. These 4 large regions of the brain were also averaged to obtain the overall degree of amyloid deposition. 


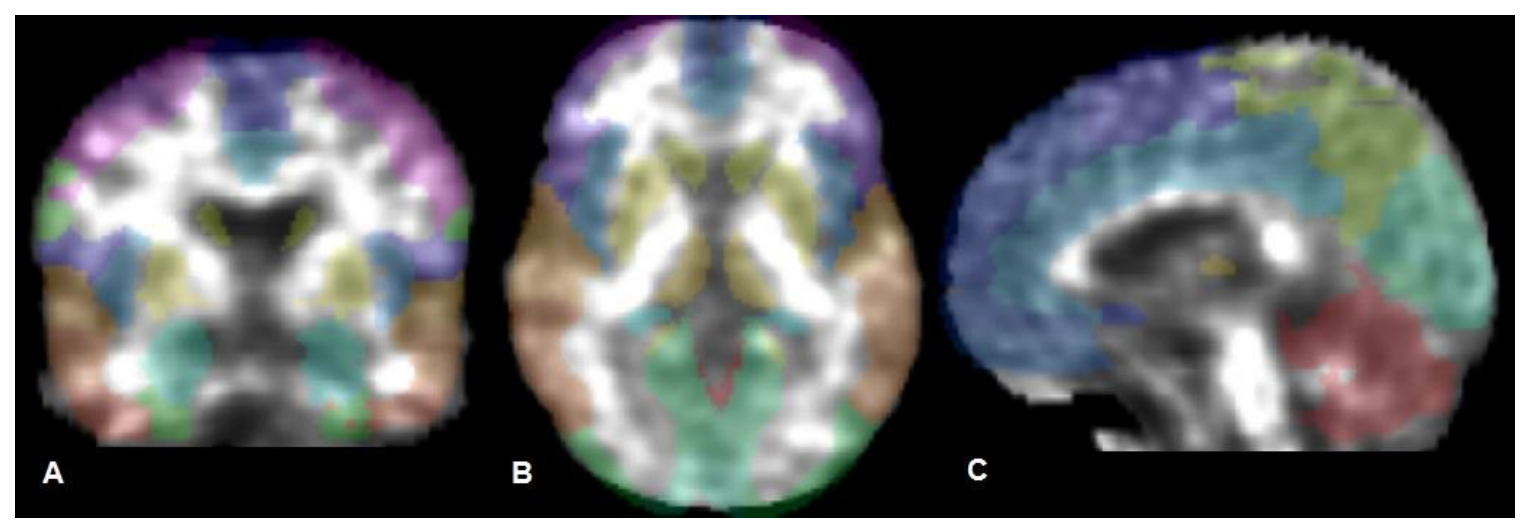

Figure 1. The Automated Anatomical Labeling atlas ${ }^{32}$ superimposed on the brain template in the coronal (A), axial (B), and sagittal (C) planes.

\subsection{Statistical analysis}

All statistical analyses were programmed in STATA version 13 (StataCorp, College Station, TX). Comparisons of baseline variables among groups were performed using the Wilcoxon rank-sum and Fisher exact tests, depending on the type and distribution of variables.

To determine the association between DM2 and brain amyloidosis, using PiB PET, we used multivariate regression analysis with amyloid deposition as the outcome variable and DM2 as the predictor variable, adjusting for age and APOE4 carrier status. Quantile-normal plots for ladder-of-powers transformations were examined, and the inverse-square transform of amyloid deposition was used for normality.

To determine the association between DM2 and cognitive deficits, we also used multivariate regression analysis. First, we determined the effect of DM2 on the Total Score from RBANS, using DM2 as the main predictor and adjusting for APOE4 carrier status. RBANS scores are already age-adjusted based on a normative database. We then determined the effects of DM2 on each RBANS subscore, as well as the Trails $A$ and Trails B subscores.

Because of the significant differences in gender proportions, amount of exercise reported, and proportion of subjects with high cholesterol in the two groups, we performed post-hoc analyses evaluating the associations of these 3 variables with brain amyloidosis and cognition.

\section{Results:}

Baseline differences between the DM2 and nondiabetic groups are shown in Table 1. The DM2 group had significantly fewer women, exercised less, and had a higher proportion of individuals with high cholesterol. Disease duration in the DM2 group ranged from $1-35$ years, with a mean duration of $15 \pm 11$ years. Recent hemoglobin A1C levels ranged from 5.7 to $14 \%$, with a mean of $8.4 \pm 2.8 \%$. Nine out of $16(56 \%)$ reported having had high glucose levels of over 300 in the past year. Thirteen were taking metformin $(81 \%)$ and four were on insulin $(25 \%)$. In addition, five reported a history of retinopathy (31\%), seven reported a history of neuropathy (44\%), and 3 reported a history of nephropathy (19\%).

Boxplots of cognitive scores by cohort are shown in Figure 2. Subjects with DM2 had significantly lower overall age-adjusted RBANS Total Scores than nondiabetics $(\mathrm{p}=0.018)$ with a difference of 11 points. Considering individual cognitive domains, DM2 subjects had significantly lower Visuospatial/Construction Scores (coefficient $=-9.0, p=$ 
0.016 ), as well as marginally lower Attention (coefficient $=-11.2, p=0.051$ ) and Language (coefficient $=-9.2, p=0.075$ ) Scores. DM2 subjects also had lower Immediate Memory (coefficient $=-5.1, p=0.33$ ) and Delayed Memory Scores (coefficient $=-4.5, p=0.21$ ), but these did not reach statistical significance. Adjusting for age and APOE4 status, subjects with DM2 scored significantly lower on the Trailmaking A test (Z-score coefficient $=-0.64, p=0.007)$ and marginally lower on the Trailmaking $B$ test (Z-score coefficient $=-0.79, p=0.076)$.

Boxplots of PiB uptake ratio, normalized by cerebellar uptake, by cohort are shown in Figure 3. Compared to nondiabetics and adjusting for age and APOE4 status, subjects with DM2 had no significant differences in overall brain amyloidosis $(p=0.25)$, frontal regions $(p=0.33)$, cingulate regions $(p=0.19)$, parietal regions $(p=0.26)$, or temporal regions $(p=0.37)$.

Post-hoc analyses demonstrated that female gender was not significantly associated with brain amyloidosis ( $p=0.27$ ), but was significantly associated with higher RBANS Total Scores (coefficient $=13.8, p=0.003$ ). Similarly, exercise was not significantly associated with brain amyloidosis $(p=0.31)$, but was significantly associated with higher RBANS Total Scores (coefficient $=1.36, p=0.019$ ). Surprisingly, hypercholesterolemia was associated with less brain amyloidosis $(p=0.039)$, but not associated with the RBANS Total Score (coefficient $=-1.1, p=0.82$ ).

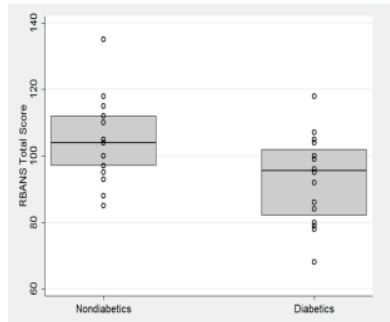

(A)

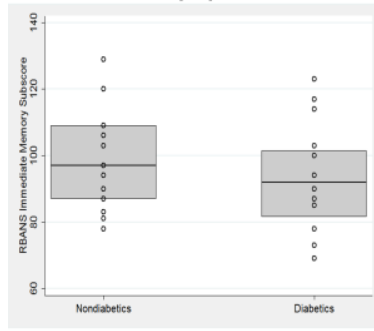

(E)

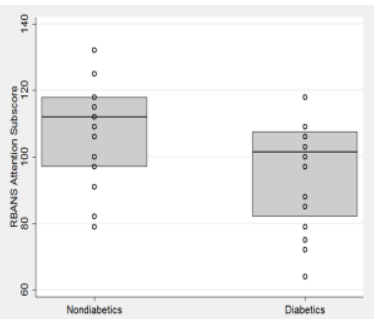

(B)

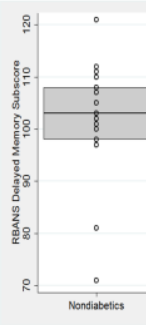

$(\mathrm{F})$

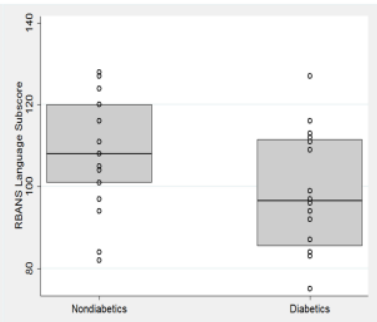

(C)

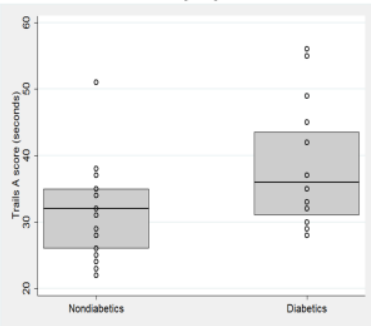

(G)

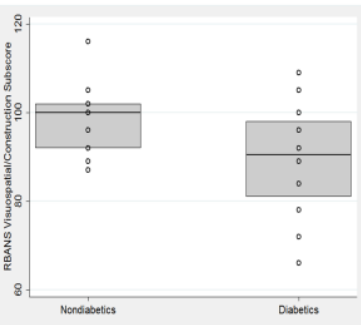

(D)

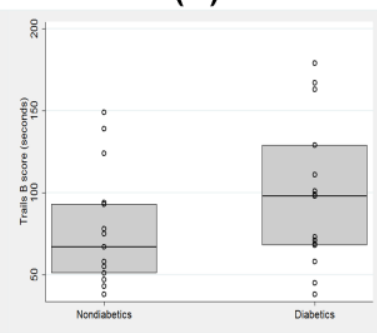

(H)

Figure 2. Boxplots of age-adjusted Total Scores (A) and Attention (B), Language (C), Visuospatial/Construction (D), Immediate Memory (E), and Delayed Memory (F) Subscores on the Repeated Battery for the Assessment of Neuropsychological Status (RBANS), by cohort. Boxplots of Trailmaking A $(\mathbf{G})$ and $B(\mathbf{H})$ Test scores also shown, by cohort. Diabetics had significantly lower Total Scores $(p=0.018)$ and

Visuospatial/Construction Subscores $(p=0.016)$ compared to non-diabetics, as well as marginally lower Attention ( $p=0.051)$ and Language $(p=0.075)$ Subscores. Diabetics also had significantly higher times on the Trailmaking $A$ test $(p=0.006)$ and marginally higher times on the Trailmaking B test $(p=0.076)$. 


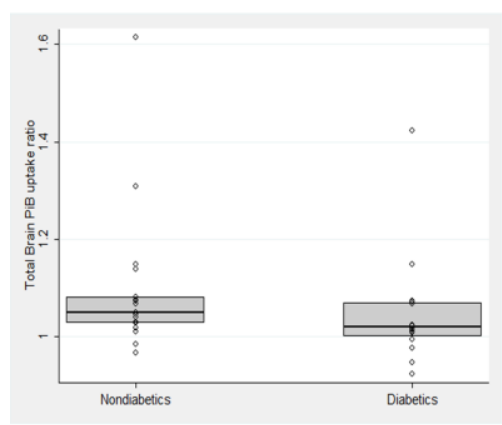

(A)

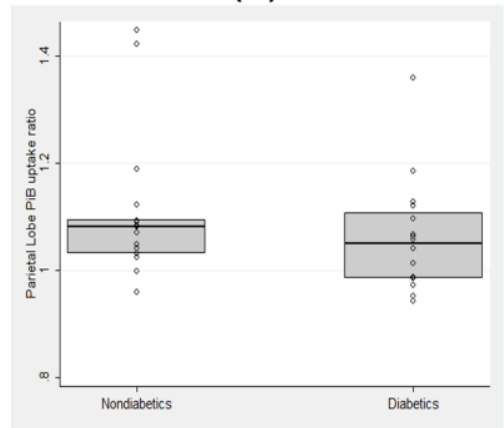

(D)

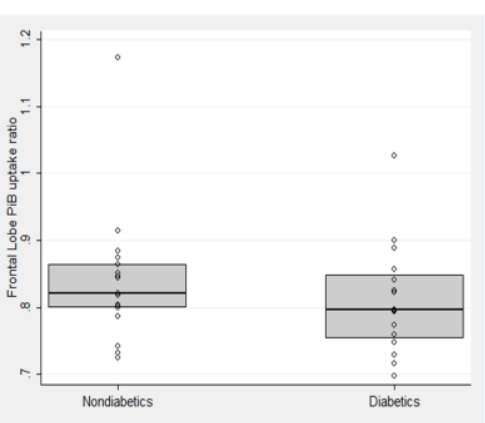

(B)

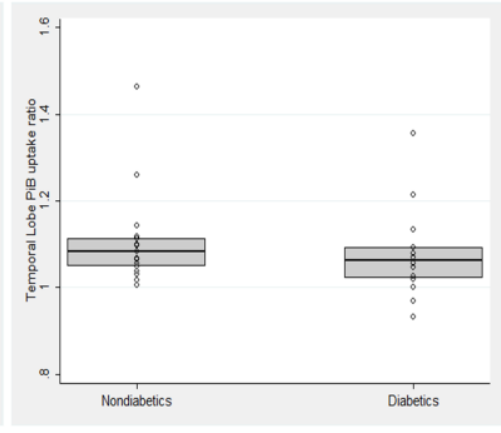

(E)

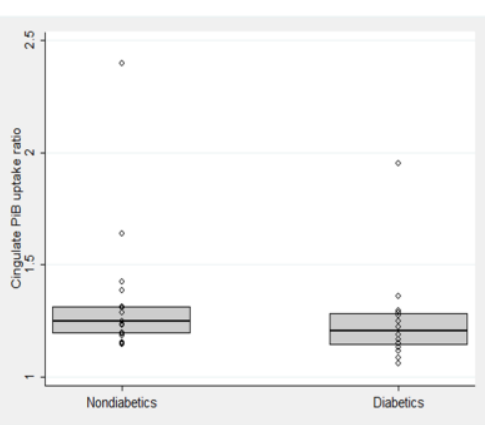

(C)

Figure 3. Boxplots of brain amyloidosis by region, measured by Pittsburgh Compound $B$ uptake on positron emission tomography. There were no significant differences in uptake between diabetics and non-diabetic cohorts in the overall brain $(p=0.25)(A)$, frontal lobes $(p=0.33)(B)$, cingulate regions $(p=0.19)(C)$, parietal lobes $(p=0.26)(D)$, or temporal lobes $(p=0.37)(E)$.

\section{Discussion:}

The goal of our study was to determine how DM2 affects brain amyloidosis and cognition in a prospective, nondemented cohort, in an effort to determine how DM2 may lead to increased risk of AD. The major findings were that 1) DM2 was not associated with increased brain amyloidosis in our cohort, 2) DM2 was associated with greater cognitive deficits, particularly in non-memory domains, and 3) these greater cognitive deficits may be mediated by differences in gender and exercise habits.

The first major finding that our DM2 cohort did not demonstrate increased brain amyloid deposition on PET was unexpected. Prior reports exploring mechanistic relationships between DM2 and amyloid have implicated insulin-degrading enzyme (IDE) as a key player, since genetic variations in IDE are related to AD risk and IDE has a role in routinely degrading amyloid as well as insulin. ${ }^{36}$ As a result, we would expect the hyperinsulinemia seen in DM2 patients to divert the enzyme towards insulin rather amyloid degradation, leading to increased brain amyloidosis. This was also described in a large autopsy study, which found increased amyloid plaques among individuals with insulin resistance, who completed a pre-mortem oral glucose tolerance test, and hyperinsulinemia. ${ }^{37}$ Another study using an animal model reported that DM2 may lead to increased amyloid deposition through advanced glycation endproducts, which aggregate with beta-amyloid plaques. ${ }^{9}$ In humans, two prior retrospective studies also showed no increase in brain amyloidosis among DM2 patients. ${ }^{13,14}$ The first study used subjects from the ADNI, ${ }^{13}$ which excluded individuals with significant vascular disease, 
using Modified Hachinski scores as exclusionary criteria, ${ }^{38}$ thereby excluding many diabetics with severe disease. The second study included a cohort in which $20 \%$ were diabetic of varying disease severity, as well as octogenarians and individuals with $\mathrm{MCl}$, who may have had increased amyloid and cognitive deficits irrespective of DM2 status. ${ }^{14}$ In our study, we specifically enrolled DM2 subjects with more severe disease - over half of our cohort had recent glucose readings over $300 \mathrm{mg} / \mathrm{dl}$, almost half had diabetic complications, the average $\mathrm{HbA} 1 \mathrm{c}$ level was over $8 \%$, and the average disease duration was 16 years. We also enrolled subjects who did not meet criteria for $\mathrm{MCl}$ or $\mathrm{AD}$, to limit effects on brain amyloidosis and cognition, irrespective of DM2. Nevertheless, our cohort of patients still did not have increased brain amyloidosis compared to non-DM2 controls, in contrast with what would be expected mechanistically. Given the findings from our study and the literature, there does not appear to be convincing evidence that individuals with DM2 have elevated risk of AD due to increased brain amyloidosis from insulin resistance. Recently, neurodegeneration without brain amyloidosis, possibly via metabolic routes, has been labeled "suspected non-Alzheimer's pathophysiology (SNAP). ${ }^{39}$ This could be a potential pathway linking DM2 and AD. An additional possibility is that our DM2 group had a higher rate of concomitant hypercholesterolemia and were therefore on HMG-CoA reductase inhibitors, which may stimulate IDE secretion and induce degradation of beta-amyloid. ${ }^{40,41} \mathrm{~A}$ larger prospective cohort study should be performed for confirmation of our findings.

Despite having no significant difference in brain amyloidosis, our DM2 subjects still had greater cognitive deficits across all domains, but most significantly in nonmemory domains. Several prior studies evaluating cognition among individuals with DM2 reported increased cognitive deficits globally, ${ }^{7}$ as well as in visuospatial/construction tasks, ${ }^{7,42}$ executive function, ${ }^{42,43}$ and processing speed. ${ }^{42,43}$ Two studies found deficits in memory among DM2 patients, ${ }^{7,42}$ although one did not. ${ }^{43}$ When we investigated potential mediators to explain these cognitive deficits, one factor that emerged could be that our DM2 subjects reported exercising less frequently. One large-scale trial and a meta-analysis reported that exercise programs significantly improve cognition, primarily executive function, ${ }^{44,45}$ although several randomizedcontrolled trials are ongoing. ${ }^{46,47}$ Because of the size of our sample, we could not perform formal subgroup analyses, although this deserves to be explored further. Another potential modifying factor is the difference in gender breakdown between our DM2 and non-DM2 patients, with more women being in the non-DM2 group. A few papers have suggested that older women may be more resistant to age-related changes than men, thereby scoring higher than men on many cognitive tests, ${ }^{48-50}$ although women decline more rapidly with onset of memory symptoms. ${ }^{51}$ Interaction between gender and DM2 may also mediate effects on cognition.

Finally, besides the possible effects of gender, exercise, and cholesterolmedication in mediating cognitive differences between DM2 and non-DM2 subjects, numerous other factors may be contributory. Prior reports have suggested roles for diabetes medications, particularly pioglitazone, ${ }^{52}$ adiponectin, ${ }^{53}$ inflammation, ${ }^{54,55}$ advanced glycation endproducts, ${ }^{56}$ tau pathology, ${ }^{13}$ and vasoreactivity. ${ }^{55}$ Amylin, another peptide that is degraded by IDE and associated with metabolic disorders, ${ }^{57}$ may be the inciting factor in AD pathogenesis, rather than amyloid. These other mediators could not be probed in our small cohort. In addition to small sample size, another limitation of our study was the cross-sectional nature, in that the patients with greater cognitive deficits would require longer follow-up to determine whether these deficits translate into higher risk of developing AD. Furthermore, longitudinal follow-up would also allow us to determine whether DM2 individuals are more likely to develop brain amyloidosis later on. Rate of family history of dementia also differed between DM2 and 
non-DM2 groups, although if family history played a role, we would expect DM2 subjects to have lower levels of brain amyloidosis and fewer cognitive deficits, which was not the case. Finally, we probed multiple vascular risk factors through medical histories, but did not image or quantify small vessel ischemic disease, since some subjects refused MRI due to claustrophobia.

\section{Conclusion:}

In conclusion, we found that community-dwelling, nondemented individuals with DM2 had greater cognitive deficits across all domains, particularly non-memory domains. Furthermore, these cognitive deficits did not appear to be mediated by brain amyloidosis. Further studies exploring the potential roles of exercise, cholesterol, and gender in mediating these cognitive deficits should be performed. 


\section{References}

1. Shaw JE, Sicree RA, Zimmet PZ. Global estimates of the prevalence of diabetes for 2010 and 2030. Diabetes Res Clin Pract 2010;87(1): 4-14.

2. Brookmeyer R, Johnson E, Ziegler-Graham K, Arrighi HM. Forecasting the global burden of Alzheimer's disease. Alzheimers Dement 2007;3(3):186-191.

3. Xu WL, von Strauss E, Qiu CX, Winblad B, Fratiglioni L. Uncontrolled diabetes increases the risk of Alzheimer's disease: a population-based cohort study. Diabetologia 2009;52(6):1031-1039.

4. Ohara T, Doi Y, Ninomiya T, Hirakawa Y, Hata J, Iwaki T, Kanba S, Kiyohara Y. Glucose tolerance status and risk of dementia in the community: The Hisayama Study. Neurology 2011;77(12):1126-1134.

5. Whitmer RA, Sidney S, Selby J, Johnston SC, Yaffe K. Midlife cardiovascular risk factors and risk of dementia in late life. Neurology 2005;64(2):277-281.

6. Ott A, Stolk RP, van Harskamp F, Pols HA, Hofman A, Breteler MM. Diabetes mellitus and the risk of dementia: The Rotterdam Study. Neurology 1999;53(9):19371942.

7. Arvanitakis Z, Wilson RS, Bienias JL, Evans DA, Bennett DA. Diabetes mellitus and risk of Alzheimer disease and decline in cognitive function. Arch Neurol 2004;61 (5):661666.

8. Hardy J, Selkoe DJ. The amyloid hypothesis of Alzheimer's disease: progress and problems on the road to therapeutics. Science 2002; 297(5580): 353-356.

9. Bitel CL, Kasinathan C, Kaswala RH, Klein WL, Frederikse PH. Amyloid-beta and tau pathology of Alzheimer's disease induced by diabetes in an animal model. $J$ Alzheimers Dis 2012;32(2):291-305.

10. Sonnen JA, Larson EB, Brickell K, Crane PK, Woltjer R, Montine TJ, Craft S. Different patterns of cerebral injury in dementia with or without diabetes. Arch Neurol 2009;66(3):315-322.

11. Yan SD, Chen X, Fu J, Chen M, Zhu H, Roher A, Slattery T, Zhao L, Nagashima M, Morser J, Migheli A, Nawroth P, Stern D, Schmidt AM. RAGE and amyloid-beta peptide neurotoxicity in Alzheimer's disease. Nature 1996;382(6593):685-691.

12. Deane R, Du Yan S, Submamaryan RK, LaRue B, Jovanovic S, Hogg E, Welch D, Manness L, Lin C, Yu J, Zhu H, Ghiso J, Frangione B, Stern A, Schmidt AM, Armstrong DL, Arnold B, Liliensiek B, Nawroth P, Hofman F, Kindy M, Stern D, Ziokovic B. RAGE mediates amyloid-beta peptide transport across the blood-brain barrier and accumulation in brain. Nat Med 2003;9(7):907-913.

13. Moran C, Beare R, Phan TG, Bruce DG, Callisaya ML, Srikanth V. Type 2 diabetes mellitus and biomarkers of neurodegeneration. Neurology 2015;85(13):1123-1130.

14. Roberts RO, Knopman DS, Cha RH, Mielke MM, Pankratz VS, Boeve BF, Kantarci K, Geda YE, Jack CR Jr, Petersen RC, Lowe VJ. Diabetes and elevated hemoglobin A1C levels are associated with brain hypometabolism but not amyloid accumulation. J Nucl Med 2014;55(5):759-764.

15. Villemagne VL, Burnham S, Bourgeat $P$, Brown B, Ellis KA, Salvado O, Szoeke C, Macaulay SL, Martins R, Maruff P, Ames D, Rowe CC, Masters CL. Amyloid beta deposition, neurodegeneration, and cognitive decline in sporadic Alzheimer's disease: a prospective cohort study. Lancet Neurol 2013;12(4)357-367.

16. Roberts RO, Geda YE, Knopman DS, Christianson TJ, Pankratz VS, Boeve BF, Vella A, Rocca WA, Petersen RC. Association of duration and severity of diabetes mellitus with mild cognitive impairment. Arch Neurol 2008;65(8):1066-1073.

17. Barnes DE, Yaffe K. The projected impact of risk factor reduction on Alzheimer's disease prevalence. Lancet Neurol 2011;10(9):819-828. 
18. Sato N, Morishita R. The roles of lipid and glucose metabolism in modulation of beta-amyloid, tau, and neurodegeneration in the pathogenesis of Alzheimer disease. Front Aging Neurosci 2015;7:199.

19. Mosconi, L, Rinne JO, Tsui WH, Berti V, Li Y, Wang H, Murray J, Scheinin N, Nagren K, Williams S, Glodzik L, De Santi S, Vallabhajosula S, de Leon MJ. Increased Fibrillar Amyloid-beta burden in normal individuals with a family history of late-onset Alzheimer's. Proc Natl Acad Sci USA 2010;107(13):5949-5954.

20. Rattanabannakit C, Risacher SL, Gao S, Lane KA, Brown SA, McDonald BC, Unverzagt FW, Apostolova LG, Saykin AJ, Farlow MR. The Cognitive Change Index as a measure of self and informant perception of cognitive decline: relation to neuropsychological tests. J Alzheimers Dis 2016;51(4):1145-1155.

21. Reisberg B, Shulman MB, Torossian C, Leng L, Zhu W. Outcome over seven years of healthy adults with and without subjective cognitive impairment. Alzheimer's Dement 2010;6(1):11-24.

22. van Oijen M, de Jong FJ, Hofman A, Koudstaal J, Breteler MM. Subjective memory complaints, education, and risk of Alzheimer's disease. Alzheimer's Dement 2007;3(2):92-97.

23. Spauwen PJ, Martens RJ, Stehouwer CD, Verhey FR, Schram MT, Sep SJ, van der Kallen CJ, Dagnelie PC, Henry RM, Schaper NC, van Boxtel MP. Lower verbal intelligence is associated with diabetic complications and slower walking speed in people with Type 2 diabetes: the Maastrict Study. Diabet Med 2016 (epub ahead of print). 24. Chin J, Madison A, Gao X, Graumlich JF, Conner-Garcia T, Murray MD, StineMorrow EA, Morrow DG. Cognition and health literacy in older adults' recall of self-care information. Gerontologist 2015 (epub ahead of print) 25. Karantzoulis S, Novitski J, Gold M, Randolph C. The Repeatable Battery for the Assessment of Neuropsychological Status (RBANS): utility in detection and characterization of mild cognitive impairment due to Alzheimer's disease. Arch Clin Neuropsychol 2013;28(8):837-844.

26. Shindo A, Terada S, Sato S, Ikeda C, Nagao S, Oshima E, Yokota O, Uchitomi Y. Trail making test part $\mathrm{A}$ and brain perfusion imaging in mild Alzheimer's disease. Dement Geriatr Cogn Dis Extra 2013;3(1):202-211.

27. Terada S, Sato S, Nagao S, Ikeda C, Shindo A, Hayashi S, Oshima E, Yokota O, Uchitomi $Y$. Trail making test $B$ and brain perfusion imaging in mild cognitive impairment and mild Alzheimer's disease. Psychiatry Res 2013;213(3): 249-255.

28. Lasa L, Ayuso-Mateos JL, Vazquez-Barquero JL, Diez-Manrique FJ, Dowrick CF. The use of the Beck Depression Inventory to screen for depression in the general population: preliminary analysis. J Affect Disord 2000;57(1-3):261-265.

29. Beck AT, Epstein N, Brown G, Steer RA. An inventory for measuring clinical anxiety: psychometric properties. J Consult Clin Psychol 1988;56(6):893-897.

30. Saykin AJ, Shen L, Foroud TM, Potkin SG, Swaminathan S, Kim S, Risacher SL, Nho K, Huentelman MJ, Craig DW, Thompson PM, Stein JL, Moore JH, Farrer LA, Green RC, Bertram L, Jack CR Jr, Weiner MW. Alzheimer's Disease Neuroimaging Initiative biomarkers as quantitative phenotypes: Genetics core aims, progress, and plans. Alzheimers Dement 2010;6(3):265-273.

31. Smith SM. Fast robust automated brain extraction. Human Brain Mapping 2002; 17(3):143-155.

32. Tzourio-Mazoyer N, Landeau B, Papathanassiou D, Crivello F, Etard O, Delcroix N, Mazoyer B, Joliot M. Automated anatomical labeling of activations in SPM using a macroscopic anatomical parcellation of the MNI MRI single-subject brain. Neuroimage 2002;15(1):273-289. 
33. Petersen RC, Aisen PS, Beckett LA, Donohue MC, Gamst AC, Harvey DJ, Jack CR Jr, Jagust WJ, Shaw LM, Toga AW, Trojanowski JQ, Weiner MW. Alzheimer's Disease Neuroimaging Initiative (ADNI): Clinical characterization. Neurology 2010;74(3):201-209. 34. Mormino EC, Kluth JT, Madison CM, Rabinovici GD, Baker SL, Miller BL, Koeppe RA, Mathis CA, Weiner MW, Jagust WJ. Episodic memory loss is related to hippocampal-mediated beta-amyloid deposition in elderly subjects. Brain 2009;132(Pt5):1310-1323.

35. Jagust WJ, Landau SM, Shaw LM, Trojanowski JQ, Koeppe RA, Reiman EM, Foster NL, Petersen RC, Weiner MW, Price JC, Mathis CA. Relationships between biomarker in aging and dementia. Neurology 2009;73(15):1193-1199.

36. Qiu WQ, Folstein MF. Insulin, insulin-degrading enzyme and amyloid-beta peptide in Alzheimer's disease: review and hypothesis. Neurobiol Aging 2006;27(2):190-198.

37. Matsuzaki T, Sasaki K, Tanizaki Y, Hata J, Fujimi K, Matsui Y, Sekita A, Suzuki SO, Kanba S, Kiyohara Y, Iwaki T. Insulin resistance is associated with the pathology of Alzheimer disease: the Hisayama study. Neurology 2010;75(9):764-770.

38. Rosen WG, Terry RD, Fuld PA, Katzman R, Peck A. Pathological verification of ischemic score in differentiation of dementias. Ann Neurol 1980;7(5):486-488.

39. Jack CR, Jr. PART and SNAP. Acta Neuropathol 2014;128(6):773-776.

40. Parikh NM, Morgan RO, Kunik ME, Chen H, Aparasu RR, Yadav RK, Schulz PE, Johnson ML. Risk factors for dementia in patients over 65 with diabetes. Int $J$ Geriatr Psychiatry 2011;26(7):749-757.

41. Son SM, Kang S, Choi H, Mook-Jung I. Statins induce insulin-degrading enzyme secretion from astrocytes via an autophagy-based unconventional secretory pathway. Mol Neurodegener 2015;10:56.

42. Moran C, Phan TG, Chen J, Blizzard L, Beare R, Venn A, Munch G, Wood AG, Forbes J, Greenaway TM, Pearson S, Srikanth V. Brain atrophy in type 2 diabetes: regional distribution and influence on cognition. Diabetes Care 2013;36(12):4036-4042. 43. Qiu C, Sigurdsson S, Zhang Q, Jonsdottir MK, Kjartansson O, Eiriksdottir G, Garcia ME, Harris TB, van Buchem MA, Gudnason V, Launer LJ. Diabetes, markers of brain pathology, and cognitive function: the Age, Gene/Environment Susceptibility-Reykjavik Study. Ann Neurol 2014;75(1):138-146.

44. Ohman H, Savikko N, Strandberg TE, Kautiainen H, Raivio MM, Laakkonen ML, Tilvis R, Pitkala KH. Effects of exercise on cognition: the Finnish Alzheimer Disease Exercise Trial: a randomized, controlled trial. J Am Geriatr Soc 2016;64(4):731-738. 45. Strohle A, Schmidt DK, Schultz F, Fricke N, Staden T, Hellweg R, Priller J, Rapp MA, Rieckmann N. Drug and exercise treatment of Alzheimer disease and mild cognitive impairment: a systematic review and meta-analysis of effects on cognition in randomized controlled trials. Am J Geriatr Psychiatry 2015;23(12):1234-1249.

46. de Souto Barreto P, Denormandie P, Lepage B, Armaingaud D, Rapp T, Chauvin P, Vellas B, Rolland Y. Effects of a long-term exercise programme on functional ability in people with dementia living in nursing homes: research protocol of the LEDEN study, a cluster randomized controlled trial. Contemp Clin Trials 2016;47:289-295.

47. Hoffmann K, Frederiksen KS, Sobol NA, Beyer N, Vogel A, Simonsen AH, Johannsen P, Lolk A, Terkelsen O, Cotman CW, Hasselbalch SG, Waldemar G.

Preserving cognition, quality of life, physical health and functional ability in Alzheimer's disease: the effect of physical exercise (ADEX trial): rationale and design.

Neuroepidemiology 2013;41(3-4):198-207.

48. McCarrey AC, An Y, Kitner-Triolo MH, Ferrucci L, Resnick SM. Sex differences in cognitive trajectories in clinically normal older adults. Psychol Aging 2016;31(2):166-175. 
49. Rolandsson O, Backestrom A, Eriksson S, Hallmans G, Nilsson LG. Increased glucose levels are associated with episodic memory in nondiabetic women. Diabetes 2008;57(2):440-443.

50. van Exel E, Gussekloo J, de Craen AJM, Bootsma-van der Wiel A, Houx P, Knook DL, Westendorp RG. Cognitive function in the oldest old: women perform better than men. J Neurol Neurosurg Psychiatry 2001;71(1):29-32.

51. Lin KA, Choudhury KR, Rathakrishnan BG, Marks DM, Petrella JR, Doraiswamy PM. Marked gender differences in progression of mild cognitive impairment over 8 years. Alzheimers Dementia 2015;1(2):103-110.

52. Heneka MT, Fink A, Doblhammer G. Effect of pioglitazone medication on the incidence of dementia. Ann Neurol 2015;78(2):284-294.

53. van Himbergen TM, Beiser AS, Ai M, Seshadri S, Otokozawa S, Au R, Thongtang N, Wolf PA, Schaefer EJ. Biomarkers for insulin resistance and inflammation and the risk for all-cause dementia and Alzheimer disease. Arch Neurol 2012;69(5):594-600. 54. Sonnen JA, Larson EB, Brickell K, Crane PK, Woltjer R, Montine TJ, Craft S. Different patterns of cerebral injury in dementia with or without diabetes. Arch Neurol 2009;66(3):315-322.

55. Chung CC, Pimental D, Jor'dan AJ, Hao Y, Milberg W, Novak V. Inflammationassociated declines in cerebral vasoreactivity and cognition in type 2 diabetes. Neurology 2015;85(5):450-458.

56. Yaffe K, Lindquist K, Schwartz AV, Vitartas C, Vittinghoff E, Satterfield S, Simonsick EM, Launer L, Rosano C, Cauley JA, Harris T. Advanced glycation end product level, diabetes, and accelerated cognitive aging. Neurology 2011;77(14):1351-1356.

57. Jackson K, Barisone GA, Diaz E, Jin LW, DeCarli C, Despa F. Amylin deposition in the brain: a second amyloid in Alzheimer disease? Ann Neurol 2013;74(4):517-526. 


\section{Acknowledgements}

We would like to acknowledge Dr. Robert Krikorian (Professor in the Department of Psychiatry \& Behavioral Neuroscience, University of Cincinnati) for his helpful suggestions and expert commentary regarding our manuscript.

Data collection and sharing for this project was funded by the Alzheimer's Disease Neuroimaging Initiative (ADNI) (National Institutes of Health Grant U01 AG024904) and DOD ADNI (Department of Defense award number W81XWH-12-2-0012). ADNI is funded by the National Institute on Aging, the National Institute of Biomedical Imaging and Bioengineering, and through generous contributions from the following: Alzheimer's Association; Alzheimer's Drug Discovery Foundation; Araclon Biotech; BioClinica, Inc.; Biogen Idec Inc.; Bristol-Myers Squibb Company; Eisai Inc.; Elan Pharmaceuticals, Inc.; Eli Lilly and Company; Eurolmmun; F. Hoffmann-La Roche Ltd and its affiliated company Genentech, Inc.; Fujirebio; GE Healthcare; ; IXICO Ltd.; Janssen Alzheimer Immunotherapy Research \& Development, LLC.; Johnson \& Johnson Pharmaceutical Research \& Development LLC.; Medpace, Inc.; Merck \& Co., Inc.; Meso Scale Diagnostics, LLC.; NeuroRx Research; Neurotrack Technologies; Novartis Pharmaceuticals Corporation; Pfizer Inc.; Piramal Imaging; Servier; Synarc Inc.; and Takeda Pharmaceutical Company. The Canadian Institutes of Health Research is providing funds to support ADNI clinical sites in Canada. Private sector contributions are facilitated by the Foundation for the National Institutes of Health (www.fnih.org). The grantee organization is the Northern California Institute for Research and Education, and the study is coordinated by the Alzheimer's Disease Cooperative Study at the University of California, San Diego. ADNI data are disseminated by the Laboratory for Neuro Imaging at the University of Southern California. 
Table 1. Baseline differences between the group with Type 2 diabetes and the non-diabetic group.

\begin{tabular}{|c|c|c|c|}
\hline & Diabetic $(n=16)$ & Nondiabetic $(n=17)$ & $P$ value \\
\hline Age (years) & $\begin{array}{l}62 \\
{[5.5]}\end{array}$ & $\begin{array}{l}63 \\
{[5.9]}\end{array}$ & 0.77 \\
\hline \% female & 19 & 59 & $0.02^{\mathrm{a}}$ \\
\hline Education (years) & $\begin{array}{l}15.4 \\
{[2.8]}\end{array}$ & $\begin{array}{l}16 \\
{[2.4]}\end{array}$ & 0.45 \\
\hline $\begin{array}{l}\% \text { with an apolipoprotein } \\
\varepsilon 2 \text { allele }\end{array}$ & 25 & 18 & 0.69 \\
\hline $\begin{array}{l}\% \text { with an apolipoprotein } \\
\varepsilon 4 \text { allele }\end{array}$ & 19 & 24 & $>0.99$ \\
\hline $\begin{array}{l}\text { \% with a family history } \\
\text { of dementia }\end{array}$ & 25 & 50 & 0.24 \\
\hline $\begin{array}{l}\text { \% with a mother with } \\
\text { Alzheimer's disease }\end{array}$ & 6 & 18 & 0.18 \\
\hline $\begin{array}{l}\text { \% with a father with } \\
\text { Alzheimer's disease }\end{array}$ & 6 & 18 & 0.60 \\
\hline \multicolumn{4}{|c|}{ Medical history and co-morbidities } \\
\hline Body mass index & $\begin{array}{l}29 \\
{[4.9]}\end{array}$ & $\begin{array}{l}26.7 \\
{[5.1]}\end{array}$ & 0.09 \\
\hline $\begin{array}{l}\text { Hours of exercise per } \\
\text { week }\end{array}$ & $\begin{array}{l}1.8 \\
{[2.5]}\end{array}$ & $\begin{array}{l}5.5 \\
{[4.5]}\end{array}$ & $0.005^{b}$ \\
\hline Smoking (years) & $\begin{array}{l}4.4 \\
{[10.4]}\end{array}$ & $\begin{array}{l}1.8 \\
{[7.3]}\end{array}$ & 0.17 \\
\hline \% with high cholesterol & 69 & 24 & $0.02^{\mathrm{a}}$ \\
\hline $\begin{array}{l}\% \text { with high blood } \\
\text { pressure }\end{array}$ & 44 & 41 & $>0.99$ \\
\hline $\begin{array}{l}\text { Number of hospital } \\
\text { emergency room visits } \\
\text { in the last year }\end{array}$ & $\begin{array}{l}0.31 \\
{[0.48]}\end{array}$ & $\begin{array}{l}0.18 \\
{[0.53]}\end{array}$ & 0.22 \\
\hline $\begin{array}{l}\text { Number of hospital } \\
\text { overnight stays in the } \\
\text { last year }\end{array}$ & $\begin{array}{l}0.25 \\
{[0.45]}\end{array}$ & $\begin{array}{l}0.12 \\
{[0.33]}\end{array}$ & 0.33 \\
\hline $\begin{array}{l}\text { Short Test of Functional } \\
\text { Health Literacy in Adults } \\
\text { (STOFHLA) score }\end{array}$ & $\begin{array}{l}35 \\
{[1.8]}\end{array}$ & $\begin{array}{l}36 \\
{[0.87]}\end{array}$ & 0.56 \\
\hline \multicolumn{4}{|l|}{ Self-reported Cognition } \\
\hline \% who reported being & 31 & 18 & 0.44 \\
\hline
\end{tabular}




\begin{tabular}{|l|l|l|l|}
\hline "forgetful" & & & \\
\hline $\begin{array}{l}\text { \% who reported } \\
\text { "difficulty remembering } \\
\text { things" }\end{array}$ & 25 & 18 & 0.68 \\
\hline Cognitive change index & 18.9 & & \\
score & {$[5.5]$} & 16.4 & 0.14 \\
\hline
\end{tabular}

Data presented are means [standard deviation]

aSignificance by the Fisher exact test

bignificance by the Wilcoxon rank-sum test 